\title{
Spectral and Dispersion Properties of Long Period Fiber Grating for Optical Communication Systems
}

\author{
V. Jain ${ }^{1}$, S. Pawar ${ }^{2 *}$, S. Kumbhaj ${ }^{1}$, P. K. Sen ${ }^{1}$ \\ ${ }^{1}$ Department of Applied Physics, S.G.S Institute of Technology \& Science, 23, Park Road Indore (M. P.) 452003, India \\ ${ }^{2}$ Department of Electronics \& Communication, Dr. A. P. J. Abdul Kalam University, Indore-Dewas Bypass Road, Arandia, \\ Indore (M. P.) 452016, India \\ *corresponding author, E-mail: spawargsits@gmail.com
}

\begin{abstract}
Present work deals with the analytical study of spectral and dispersion properties of long period fiber grating (LPFG) under linear regime. The standard parameters in the understanding of the optical features of an LPFG have been analyzed using the linear coupling processes such that one can appreciate without going through the cumbersome mathematical treatment of coupled mode equations the basic characteristics of the grating. We have analyze transmittance, phase factor, group delay and group velocity dispersion (GVD) of the LPFG as functions of physical parameters like operating wavelength, grating length, induced index change, and detuning parameter. Special attention is paid to the study of GVD with second and third order dispersion contribution as well as the filter characteristics and delay response of the grating. In case of strong grating, we find that at a particular grating strength the resonance band splits into two bands. Negative group delay for certain values of coupling strength suggested that an LPFG can also be used as dispersion compensator in optical fiber communication.
\end{abstract}

\section{Introduction}

In the era of light wave technology, long period fiber gratings (LPFGs) have attracted a great deal of interest because of their importance in designing new optical devices for future need of optical communication and sensing systems. An intense research work is available in the literature to investigate the possibility of the grating based devices for all optical ultrafast applications.

Many interesting research activities related with long period grating in the fields of photonics and sensors have been found in the literature. For the first time Vengsarkar et al. [1] proposed a new class of fiber grating called long period fiber grating (LPFG) that functions as spectrally selective loss element and acted as inline, low-loss and band-rejection filter. At present, long period fiber gratings are utilized in various applications such as gain flattening filters for erbium doped fiber amplifiers [2], dispersion compensator [3], add drop multiplexer [4], optical fiber polarizer [5], strain, temperature sensors [6]. The most significant properties of the LPFG is its tunability for the desired transmission characteristics by changing the grating parameters like refractive index, modulation depth, grating length, period etc.

Various mechanism has been proposed for fabrication of LPFGs using ultraviolet (UV) irradiation [7], ion implantation [8], irradiation by femto second pulses in the infrared [9], irradiation by $\mathrm{CO} 2$ lasers [10], diffusion of dopants into the core [11], relaxation of mechanical stress and electrical discharges $[12,13]$. Also, many researchers have reported their works on long period grating structure in different types of optical fiber including photonic crystal fibers [14], polymer optical fibers [15] and even chalcogenide optical fibers [16] to study their spectral characteristics at various physical conditions. Such gratings implemented using a range of different approaches, including micro-bend grating [17] and acoustic gratings [18] that are completely reconfigurable.

Zhao and Palais investigated coherence spectrum properties of long period fiber Bragg grating using mode selection rule and coupling equations [19]. Navruz and Altuncu proposed multiband rejection filter based on the optimization of a periodically phase shifted long period grating [20]. They reported that spectral position and bandwidth of each reflection band can be controlled by varying the grating length and the period. Jeong and $\mathrm{Oh}$ analyzed theoretically transmission spectrum of long period grating for an optical fiber whose material dispersion in the cladding region was modified by doping a transition metal $\mathrm{Cr}^{3+}$ [21]. The doping metal ion change the effective indices of cladding modes and subsequently the phase matching condition for coupling with the core modes in a long period fiber grating. This technique is applicable in novel filters for a wide band optical communication system. Bai and Chiang analyzed the transmission spectra of a coupler consisting of two parallel uniform or non-uniform long period waveguide grating (LPWG) using coupled mode theory [22]. Patrick et al. demonstrated the change in wavelength of a long period fiber grating attenuation band with changes in external index of refraction which can be enhanced by proper selection of the grating period. The changes in the spectrum over a wavelength range from 1100 to $1600 \mathrm{~nm}$ and $1<\mathrm{n}$ $<1.72$ index range are also presented [23]. Chiang et al. reviewed on the development of long-period fiber grating 
devices for application in optical communication. In this study they highlighted the realization of long-period gratings in optical planar waveguides [24]. Rastogi and Chiang presented a theoretical analysis of light propagation in a four-layer planar waveguide that consists of long period grating having a period of the order of $100 \mu \mathrm{m}$ [25]. Using the coupled-mode theory, they have shown such structure is capable of coupling light from the fundamental guided mode to the cladding modes at a specific wavelength (resonance wavelength) and thus results in sharp rejection bands in the transmission spectrum of the waveguide. Their numerical results show that the resonance wavelengths as well as the transmission spectrum can be significantly changed with the waveguide and grating parameters.

Deparis et al. have fabricated band pass filters based on $\pi$-shifted long-period gratings for application in actively mode-locked erbium fiber lasers. The Introduce of $\pi$-phase shift in the middle of the grating opens a band pass within the core-cladding mode resonance peaks. These all-fiber filters have the advantages of low insertion loss $(<0.5 \mathrm{~dB})$ and a wide bandwidth $(10-20 \mathrm{~nm})$ [26]. Gu and Chiang have explored the effects of average index variation on the transmission characteristics of an index-apodized longperiod fiber grating (LPFG) by the transfer matrix method and studied that how these effects depends up on the grating length, grating profile, modal dispersion factor, and the duty cycle of the index modulation [27]. M. Das and K. Thyagarajan have proposed the use of uniform LPG fabricated on relatively high refractive index difference fibers as efficient dispersion compensator [28]. They have shown that such compensators have high dispersion values with reasonable pulse compression ratio with negligible delay ripple. The proposed device has a very simple structure, low insertion loss, negligible delay ripple and is suitable for optical communication link.

The objective of present work is to describe the theoretical study of spectral characteristics and dispersion compensation properties of LPFG. Though several methods have been adopted by the researchers to study LPFG characteristics, the coupled mode theory is most popular and simplest among them [29-33]. In this paper the coupled mode theory is used and according to this theory the field amplitude for co-propagating core and cladding modes has been derived considering the propagation of a steady state quasi $\mathrm{CW}$ beam under linear regime. Analytical expression of transmission coefficient, phase and delay response of the grating have been obtained. Throughout this paper, our notation follows most closely that of G. P Agrawal [34]

This work entirely covers the fundamental optical properties of long period fiber grating and its applications in optical communication system for high speed modern photonic technology. The work in this paper is organised as follows: Section II, describe the mathematical method to solve coupled mode equation. This section provides a complete analytical treatment to describe the quasi $\mathrm{CW}$ light propagation in periodic structure. The filter characteristics of LPFGs in transmission mode are investigated in Section III. The estimation of LPFG bandwidth and its variation with various physical parameters is described in Section IV. Section V gives a brief description of optical phase response of grating for different values of grating strength. In Section VI, the grating induced dispersive properties are examined. Also the group delay and group velocity dispersion are calculated to examine the dispersion compensation capabilities of LPFGs.

\section{Mathematical Analysis}

Long-period gratings can be considered as a special class of fibre Bragg gratings in which the period of the index modulation is such that it satisfies a phase matching condition between the fundamental core mode and a forward propagating cladding mode of an optical fibre. For an LPFG, the periodic modulation of the index of refraction in the fibre core has a period typically in the range of $100 \mu \mathrm{m}$ to $1000 \mu \mathrm{m}$. The presence of modulation along the length of LPFG causes the coupling between the co-propagating core mode and specific cladding mode. This results in a series of attenuation bands centered at discrete wavelengths in the transmission spectrum. Each attenuation band is corresponding to the coupling of fundamental core mode to a different cladding mode. The interaction of one mode of a fiber with other modes is commonly described with the help of coupled-mode theory in which only two modes are supposed to be nearly phase-matched and capable of resonant coupling. Based on this theory, the phase matching condition is:

$$
\beta_{\text {core }}-\beta_{\text {clad }}^{m}=\frac{2 \pi}{\wedge}
$$

here, $\Lambda$ is the grating period of LPFG. $\beta_{\text {core }}, \beta_{c l a d}^{m}$ are the Propagation constant of fundamental core mode and for the $\mathrm{m}^{\text {th }}$ cladding mode of LPFG, respectively and are given as

$$
\beta_{\text {core }}=\frac{2 \pi n_{\text {eff }}^{\text {core }}}{\lambda_{R}} \quad \text { and } \quad \beta_{\text {clad }}^{m}=\frac{2 \pi n_{\text {eff }}^{\text {clad }(m)}}{\lambda_{R}} .
$$

Whereas, $\lambda_{R}$ is the resonance wavelength of the resonance band. $n_{\text {eff }}^{\text {core }}, n_{\text {eff }}^{\text {clad }(m)}$ are the effective refractive indices of core and $\mathrm{m}^{\text {th }}$ cladding modes, respectively. Equation (1) and (2) leads the phase matching condition as

$$
\lambda_{R}=\left(n_{\text {efff }(c o)}-n_{\text {eff }(c l)}^{m}\right) \Lambda=\Delta n_{\text {eff }} \Lambda .
$$

In the above Equation, $\Delta n_{\text {eff }}$ is the difference in the effective indices of refraction of the core and cladding modes.

We assume that the fiber is lossless and single mode in the wavelength range of interest. Moreover, we assumed that the fiber is weakly guiding, i.e. the difference between the refractive indices in the core and the cladding is very small, as well as the electric and magnetic fields are approximately transverse to the fiber axis, thus we can ignore all polarization effects due to the fiber structure and consider solely the scalar wave equation.

According to the coupled mode theory, the total field at any value of $z$ can be written as a superposition of the two interacting modes and the coupling process results in $\mathrm{z}-$ dependent amplitude of the two coupled modes. It is 
assumed that the long period grating within the single-mode fiber has a forward propagating core mode and a co propagating cladding mode. Thus, the total electric field within the grating is given as

$$
\vec{E}(r, \omega)=\left(\vec{E}_{\text {core }}(r, \omega)+\vec{E}_{\text {clad }}(r, \omega)\right)
$$

here, $\vec{E}_{\text {core }}(r, \omega)=\vec{A}_{\text {core }}(z, \omega) F_{\text {core }}(x, y) \exp \left(i \beta_{\text {core }} z\right)$

and $\vec{E}_{\text {clad }}(r, \omega)=\vec{A}_{\text {cladd }} F_{\text {clad }}(z, \omega) \exp \left(i \beta_{\text {clad }} z\right)$.

In the above equations $A_{\text {core }}, F_{\text {core }}(x, y)$ and $A_{\text {clad }}, F_{\text {clad }}(x, y)$ represents the slowly varying amplitudes, the transverse modal field distribution function for two co- propagating core and cladding modes coupled by the grating, respectively. In order to examine the characteristics of the co- propagating core and cladding modes coupled by the grating, following pair of linear coupled mode equations (LCMEs) have been used. [34, 35]

$\frac{\partial A_{\text {core }}}{\partial z}+\frac{1}{V_{g 1}} \frac{\partial A_{\text {core }}}{\partial t}+\frac{i \beta_{21}}{2} \frac{\partial^{2} A_{\text {core }}}{\partial t^{2}}=i \delta A_{\text {core }}+i \kappa A_{\text {clad }}$

and

$\frac{\partial A_{\text {clad }}}{\partial z}+\frac{1}{V_{g 2}} \frac{\partial A_{\text {clad }}}{\partial t}+\frac{i \beta_{22}}{2} \frac{\partial^{2} A_{\text {clad }}}{\partial t^{2}}=-i \delta A_{\text {clad }}+i \kappa A_{\text {core }}$

Here $\beta_{11}=1 / \mathrm{V}_{\mathrm{g} 1}, \beta_{12}=1 / \mathrm{V}_{\mathrm{g} 2}$ and $\beta_{21}, \beta_{22}$ are first and second order dispersion parameters related to the group velocity. These parameters can be different for the two modes because of their different mode indices. $\delta$ and $\kappa$ are detuning parameter and linear coupling coefficient for the grating, respectively, and are defined as

$$
\delta=\frac{1}{2}\left(\beta_{\text {core }}-\beta_{\text {clad }}^{m}-\frac{2 \pi}{\wedge}\right), \text { and } \quad \kappa=\frac{\pi n_{g}}{\lambda_{R}} .
$$

Where $n_{g}$ is induced modulation in the refractive index of core arising while grating in the fiber is written. In the steady state $\mathrm{CW}$ linear regime, dispersion parameters are neglected. With these assumptions LCMEs take the simplified forms as,

$$
\begin{aligned}
& \frac{\partial A_{\text {core }}}{\partial z} & =i \delta A_{\text {core }}+i \kappa A_{\text {clad }} \\
\text { and } \quad & \frac{\partial A_{\text {clad }}}{\partial z} & =-i \delta A_{\text {clad }}+i \kappa A_{\text {core }}
\end{aligned}
$$

Differentiation of equations (10) and (11) with respect to $\mathrm{z}$ and further simplification provides

$$
\frac{\partial^{2} A_{\text {core }}}{\partial z^{2}}+q^{2} A_{\text {core }}=\mathbf{O}
$$

and

$$
\frac{\partial^{2} A_{\text {clad }}}{\partial z^{2}}+q^{2} A_{\text {clad }}=\mathbf{O} \text {. }
$$

Here parameter $\mathrm{q}$ is the dispersion parameter of long period fiber grating given by the dispersion relation

$$
q=\sqrt{\delta^{2}+\kappa^{2}}
$$

General solutions of the Equations (12) and (13) are obtained as

$$
A_{\text {core }}(z)=C_{1} e^{i q z}+C_{2} e^{-i q z}
$$

and

$$
A_{\text {clad }}(z)=D_{1} e^{i q z}+D_{2} e^{-i q z}
$$

The above equations show that, the $\mathrm{z}$ dependent parts of copropagating core and cladding modes in LPFG varies exponential with the propagation constant q. The constant $\mathrm{C}_{1}, \mathrm{C}_{2}, \mathrm{D}_{1}$ and $\mathrm{D}_{2}$ in Equations (15) and (16) are interdependent and satisfy following four relations:

$$
\begin{aligned}
& (q-\delta) C_{1}=\kappa D_{1} ;(q+\delta) C_{2}=-\kappa D_{2} \\
& (q+\delta) D_{1}=\kappa C_{1} ;(q-\delta) D_{2}=-\kappa C_{2} .
\end{aligned}
$$

Using Equations (17), (18) in Equations (15), (16), the general solutions for amplitudes of core and cladding modes can be written in terms of an effective transmission coefficient $\mathrm{t}(\mathrm{q})$ as

$$
\begin{aligned}
& A_{\text {core }}(z)=D_{1} e^{i q z} t(q)+C_{2} e^{-i q z} \\
& A_{\text {clad }}(z)=D_{1} e^{i q z}-C_{2} e^{-i q z} t(q)
\end{aligned}
$$

here,

$$
t(q)=\left(\frac{\kappa}{q+\delta}\right)=\left(\frac{q-\delta}{\kappa}\right)
$$

The q dependent transmission coefficient (Eq. 21) and the dispersion relation (Eq.14) indicate that both the magnitude and phase of the transmitted wave depends upon the frequency $\omega$ of propagating beam in the LPFG.

\section{Filter Response of Long Period Fiber Grating}

To study the filter response of LPFG, we have determined transmission coefficient ( $\left.t_{\mathrm{LPFG}}\right)$ of LPFG by applying boundary condition that light is incident only at the front end at $\mathrm{z}=0$ of LPFG i.e.,

$$
A_{\text {core }}(z=0)=1 \text {, and } A_{\text {clad }}(z=0)=0
$$

Using equation (22) in equation (20) we find

$$
C_{2}=\frac{D_{1}}{t(q)} \text {. }
$$

The transmission coefficient of the long period fibre grating is define as the ratio of amplitudes of the core mode at $z=L$ and at $\mathrm{z}=0$. Thus the transmission coefficient,

$$
t_{\text {LPFG }}=\frac{A_{\text {core }}(z=L)}{A_{\text {core }}(z=0)}=\frac{q \cos (q L)+i \delta \sin (q L)}{q} .
$$

The corresponding expression for the transmittance in the linear regime is found as

$$
T_{L P F G}=\left|t_{L P F G}\right|^{2}=\frac{\delta^{2}+\kappa^{2} \cos ^{2} q L}{q^{2}} .
$$

At resonance $\delta=0$, the transmission is minimum for core mode and given as

$$
\left(T_{L P F G}\right)_{\min }=\cos ^{2} \kappa L .
$$

Also, the maximum cross transmission $T^{x}{ }_{L P G}$ i.e. the fraction of energy transmitted to the cladding mode is found to be

$$
\left(T_{L P F G}^{x}\right)_{\max }=\sin ^{2} \kappa L .
$$

Using equation (25), the transmittance of the grating is plotted in Fig. 1 as a function of wavelength for different grating lengths such as (1) $\mathrm{L}=0.25 \mathrm{~cm}$, (2) $\mathrm{L}=0.5 \mathrm{~cm}$, (3) $\mathrm{L}=1.0 \mathrm{~cm}$, (4) $\mathrm{L}=1.5 \mathrm{~cm} \mathrm{(5)} \mathrm{L}=2.0 \mathrm{~cm}$ and (6) $2.5 \mathrm{~cm}$. We have considered silica glass LPFG with physical 
parameters, $\mathrm{n}_{\text {core }}=1.458$, cladding index $\mathrm{n}_{\text {clad }}=1.450, \mathrm{n}_{\mathrm{g}} \approx$ $1 \times 10^{-4}, \Lambda=197.5 \mu \mathrm{m}$ and $\lambda_{\mathrm{R}} \approx 1550 \mathrm{~nm}$.

To study the transmission response of grating first we consider the grating coupling strength $\kappa L \leq 1$ as shown in Fig. 1. In this coupling strength long period fiber grating shows "sinc" shaped dip in transmitted spectrum at resonance wavelength, whose bandwidth is inversely proportional to the grating length and depth of transmitted dip depends upon the value of $\kappa$, but the overall spectral shape and bandwidth is determined only by the grating length L.

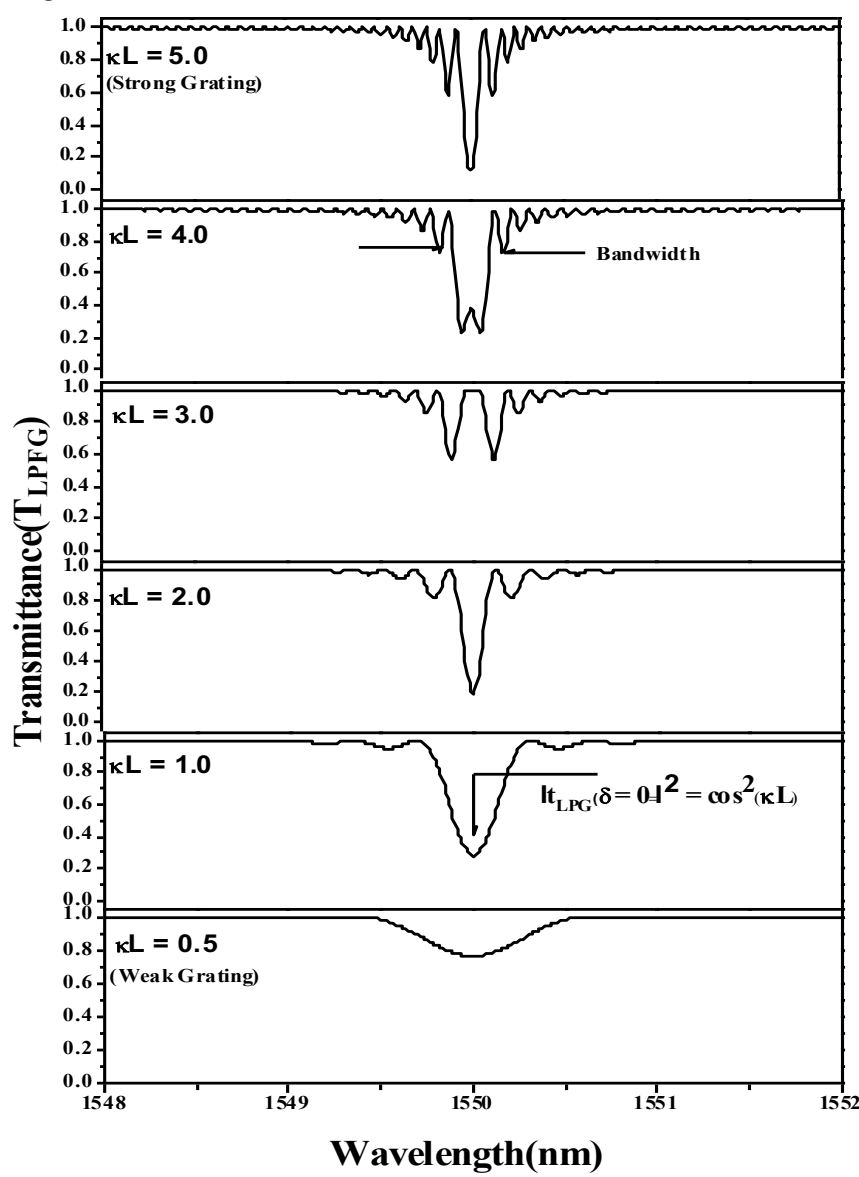

Figure 1: Calculated transmission response as a function of wavelength for six different long period grating structures with increasing lengths.

The grating design at $\kappa L \leq 1$ it does not make a suitable band rejection filter, because it partially transmits the input signal. However, there are number of cases where the "sinc" shaped spectral response is desirable this type of designed grating is very useful. In many binary communications systems, the encoded signal has precisely at the same "sinc"shaped spectral response. If we consider the grating coupling strength $\kappa L \geq 1$, the spectral response has one or two dips with very narrow band, as shown in Fig. 1. At this coupling strength, grating shows very low transmittance within a band of frequencies can be called as the attenuation band. Outside of the band, the spectrum shows a series of undesirable ripples or side lobes. These side lobes originate at the two grating ends where the refractive index changes suddenly as compared to its value outside the grating region. These lobes decrease as we move away from the resonance wavelength until the structure is effectively transparent. If the grating is made longer without changing the value of $\kappa$, the bandwidth of attenuation band remains unchanged, but spectrum splits into one or two dips and the side lobes get closer together.

The minimum transmittance $\left(\mathrm{T}_{\mathrm{LPFG}}\right)_{\mathrm{min}}$ at the resonance wavelength $\lambda_{R}$ is the most important parameter of interest to design long period fiber grating.

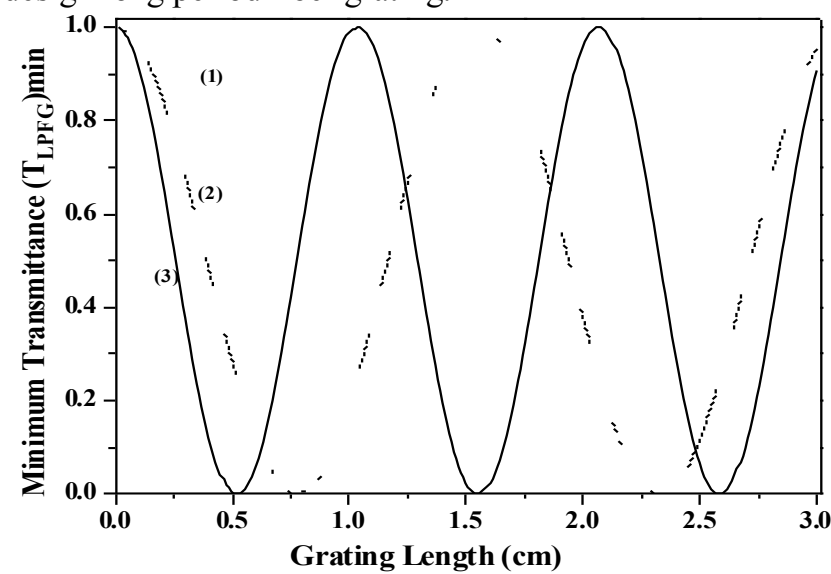

Figure 2: Minimum transmittance as a function of grating length (L), calculated for different values of grating index (1) $0.5 \times 10^{-4}$ (dotted curve), (2) $1.0 \times 10^{-4}$ (dashed curve) and (3) $1.5 \times 10^{-4}$ (solid curve).

Fig. 2 shows the dependence of minimum transmittance (TLPFG) min on the grating length (L) and refractive index change $\left(n_{g}\right)$. It is clear, that the minimum transmittance at resonance wavelength vary as a cosine function with length. Also it is observed that the same minimum transmittance can be achieved with shorter gratings using high $n_{g}$ values. Hence proper selection of physical parameter of long period fiber grating enable us to utilize this device as a single/double band rejection filter at a particular wavelength/band in optical communication system.

\section{Estimation of bandwidth of LPFG}

Many applications of long-period fiber gratings are based on the narrow band rejection filtering, for which the transmission bandwidth is a critical parameter. A useful measure of this bandwidth is the separation between the first two zeros of grating spectrum. For a uniform long period grating in which at most one complete exchange of power between the two modes occurs, the transmission bandwidth $\Delta \lambda$ is defined as [29]

$$
\Delta \lambda=\frac{2 \lambda_{R}^{2}}{\Delta n_{e f f} L} \sqrt{1-\left(\frac{\kappa L}{\pi}\right)^{2}} .
$$

Whereas, $\kappa \mathrm{L} \leq \pi$ and for a given value of minimum transmittance $\left(\mathrm{T}_{\mathrm{LPFG}}\right)$ min. The transmission bandwidth is calculated from equation (26). For strong gratings, undesirable side lobes become significantly more pronounced and hence, a better measure of the bandwidth is 
the FWHM of the envelope traced by the peaks of the side lobe. The calculated values for bandwidth of attenuation band of LPFGs are shown in Table 1.In equation (28) value of $\Delta \mathrm{n}_{\text {eff }}$ depends upon effective indices of core and cladding modes .These effective indices are determined using twolayer fiber geometry of step index fiber proposed by vengsarkar et.al [1]. The values of remaining physical parameter are same as considered in previous section.

Table 1: Calculated bandwidth of a long period fiber grating at different values of $\kappa L$.

\begin{tabular}{ccccc}
\hline S.No. & $\begin{array}{c}\text { LPFG } \\
\text { Length } \\
(\mathrm{cm})\end{array}$ & $\begin{array}{c}\kappa \mathrm{L} \\
(\text { approx })\end{array}$ & $\begin{array}{c}\text { Minimum } \\
\text { Transmittance } \\
(\%)\end{array}$ & $\begin{array}{c}\text { Bandwidth } \\
(\mathrm{nm})\end{array}$ \\
\hline 1 & 0.25 & 0.5 & 76 & 1.30 \\
2 & 0.5 & 1.0 & 28 & 0.60 \\
3 & 1.0 & 1.5 & 19 & 0.20 \\
4 & 1.5 & 2.0 & 98 & 0.05 \\
5 & 2.0 & 2.5 & 37 & 0.30 \\
6 & 2.5 & 3.0 & 12 & 0.16 \\
\hline
\end{tabular}

Table 1 reveals that we can obtained a desired bandwidth of long period grating by choosing appropriate values of grating length. It is important to note that for a given value of minimum transmittance ( $\left.\mathrm{T}_{\mathrm{LPG}}\right)_{\mathrm{min}}$ of long period grating and band width $\Delta \lambda$, grating length can be determined using Equation (26) and (28). And we can design a fiber grating as per the need of specific optical communication system.

\section{Phase Response of Long Period Grating}

In previous sections we have estimated the amplitude and bandwidth of long period grating. For complete characterization of the LPFG, it is also essential to know the phase response of the grating.

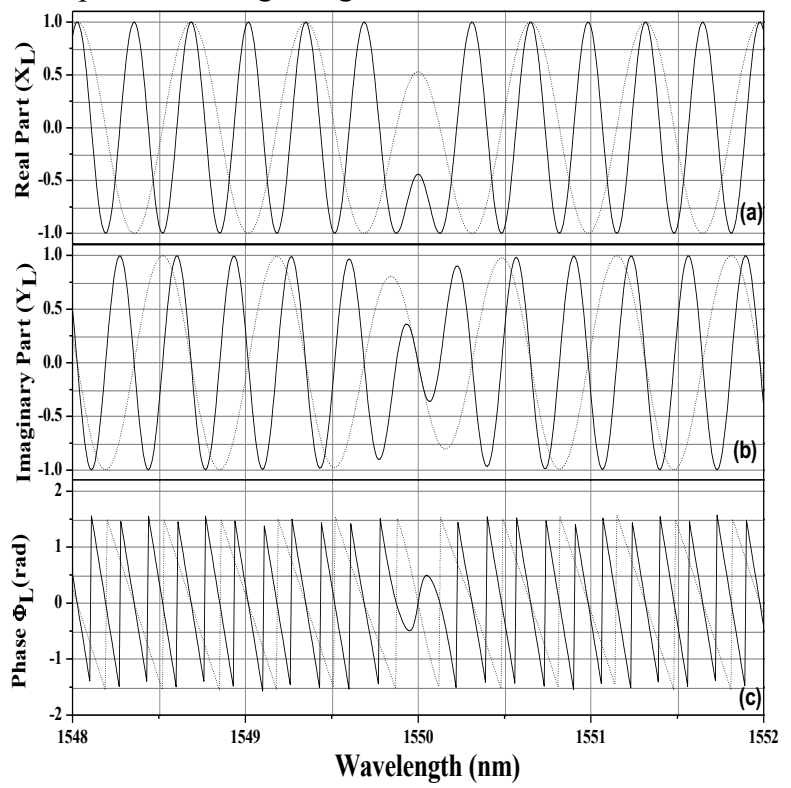

Figure 3: Variation in (a) The real part $\left(\mathrm{X}_{\mathrm{L}}\right)(\mathrm{b})$ the imaginary part $\left(Y_{L}\right)$ and $(c)$ the phase factor $\left(\phi_{\mathrm{L}}\right)$ of transmission coefficient $\left(t_{L P G}\right)$ plotted as a function of wavelength for different grating strength $\kappa \mathrm{L}$ $=1$ (dotted curve) and $\kappa \mathrm{L}=2$ (solid curve).
From equation (24), it is observed that the transmission coefficient of LPFG can be written in phasor form as [36]

$$
t(\omega)=\left|t_{L P G}\right| \times \exp \left[i \phi_{L}(\omega)\right] .
$$

Where $\left|t_{L P G}\right|$ and $\phi_{L}(\omega)$ are amplitude and phase of LPFG. On arranging the equation (24) in the form of $Z=X_{L}+i Y_{L}$, we get

$$
\left|t_{L P G}\right|^{2}=T_{L P G}=\cos q L+i \frac{\delta}{q} \sin q L .
$$

Here $X_{L}=\cos q L$ and $Y_{L}=\frac{\delta}{q} \sin q L$ are representing the real and imaginary part of the complex transmission coefficient. This gives phase factor of the transmitted wave at a distance L of LPFG as

$$
\phi_{L}(\omega)=\tan ^{-1}\left(\frac{Y_{L}}{X_{L}}\right)=\tan ^{-1}\left(\frac{\delta}{q} \tan (q L)\right) .
$$

The variation of real part, imaginary part and phase of transmission coefficient as a function of the wavelength for two different grating strength $\kappa L=1$ and $\kappa L=2$ is shown in Figure 3. This Figure revels that at outside of the attenuation band, the variation in the phase of the transmitted wave is just like unperturbed fiber core and inside the attenuation band, the phase decreases slowly with increasing strength of the grating.

\section{Delay and Dispersion Properties of LPFG}

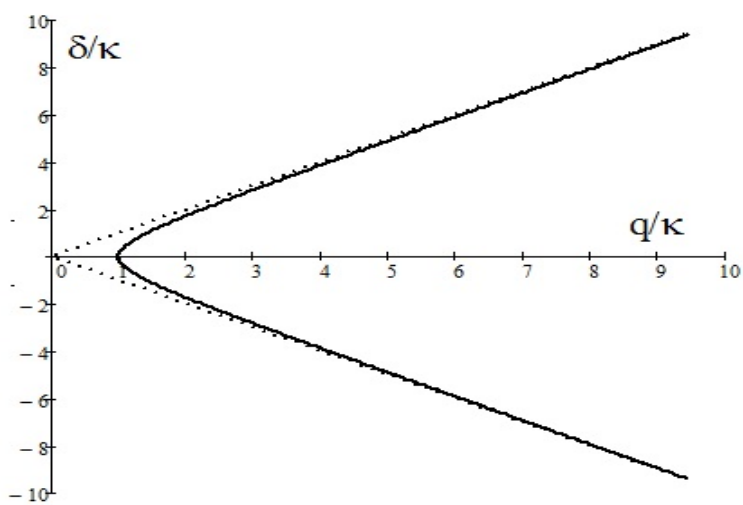

Figure 4: Dispersion curves showing variation of dispersion parameter q with detuning parameter $\delta$.

As reported earlier [37] long period fiber grating exhibits an important property known as the dispersion property, which can be seen in Figure 4. In this figure detuning parameter $\delta$ given by equation (14) is plotted as a function of dispersion parameter $\mathrm{q}$ for both uniform medium (dashed line) and a periodic medium (solid line). Since the slope is constant in case of uniform medium thus the dispersion is negligible. This dispersion is modified on introducing a grating in the fiber. If the dispersion parameter lies in the range of $0<\mathrm{q} / \kappa<1$ the core and cladding modes are coupled by the grating and hence these modes are attenuated. To understand the dispersive effect of long period fiber grating, we expand effective propagation constant of grating $\left(\beta_{\mathrm{e}}\right)$ in a Taylor series around the carrier frequency $\left(\omega_{0}\right)$ of the pulse as [34] 


$$
\beta_{e}(\omega)=\beta_{0}^{g}+\left(\omega-\omega_{0}\right) \beta_{1}^{g}+\frac{1}{2}\left(\omega-\omega_{0}\right)^{2} \beta_{2}^{g}+\frac{1}{6}\left(\omega-\omega_{0}\right)^{3} \beta_{3}^{g}+\ldots .
$$

here,

$$
\beta_{m}^{g}=\frac{d^{m} q}{d \omega^{m}} \approx\left(\frac{1}{v_{g}}\right)^{m} \frac{d^{m} q}{d \delta^{m}}
$$

Is referred as $\mathrm{m}^{\text {th }}$ order (with $\mathrm{m}=1,2,3 \ldots$ ) dispersion parameter. And $v_{g}$ is the group velocity of pulse in the absence of grating $(\kappa=0)$. In the present work we have analyzed first, second and third order dispersion parameters.

Using equation (33) and dispersion relation equation (14) the group velocity of the wave inside the grating is calculated as

$$
V_{G}=\frac{1}{\beta_{1}^{g}}=\frac{1}{\left(\frac{1}{v_{g}}\right) \frac{d q}{d \delta}}=v_{g} \sqrt{1+\frac{\kappa^{2}}{\delta^{2}}}
$$

Where, $\beta_{1}^{g}$ is the first order dispersion parameter .From equation (34), it is clear that for $\delta \rightarrow \kappa, V_{\mathrm{G}}=\sqrt{2} \mathrm{~V}_{\mathrm{g}}$ with the group velocity of pulse inside the grating become $\sqrt{2}$ times of the group velocity of pulse outside the grating. The second order dispersion parameter $\beta_{2}^{g}$ represents dispersion of group velocity and hence also called group velocity dispersion (GVD) parameter. This parameter is calculated as

$$
\beta_{2}^{g}=\left(\frac{1}{v_{g}}\right)^{2} \frac{d^{2} q}{d \delta^{2}}=\frac{\operatorname{sgn}(\delta) \frac{\kappa^{2}}{v_{g}{ }^{2}}}{\left(\delta^{2}+\kappa^{2}\right)^{\frac{3}{2}}} .
$$

The grating induced group velocity dispersion is shown in Figure 5, where GVD parameter $\beta_{2}^{g}$ is plotted as a function of wavelength $(\lambda)$. The GVD exhibits two regimes, first for wavelength $\lambda<\lambda_{R}$ and $\beta_{2}^{g}>0$, here the long period grating is said to exhibit normal dispersion and second for wavelength $\lambda>\lambda_{R}$ and $\beta_{2}^{g}<0$, where the long period grating exhibit anomalous dispersion. In the normal dispersion regime, high-frequency (blue-shifted) components of an optical pulse travel slower than lowfrequency (red-shifted) components of the same pulse. The opposite occurs in the anomalous dispersion regime where, low-frequency (red-shifted) components of an optical pulse travel slower than high-frequency (blue-shifted) components of the same pulse. It may be noticed from the figure that under the normal dispersion regime, GVD is higher for smaller coupling coefficient. While under anomalous dispersion regime behavior is opposite.

The third order dispersion in an LPFG given by $\beta_{3}^{g}$. Though it's contribution is very small as compared to $\beta_{2}^{g}$. But For ultra-short pulse propagation, it is necessary to include the $\beta_{3}$ term even when $\beta_{2} \neq 0$. Using equation (33), this parameter is obtained as

$$
\beta_{3}^{g}=\frac{d^{3} q}{d \omega^{3}} \approx\left(\frac{1}{v_{g}}\right)^{3} \frac{d^{3} q}{d \delta^{3}}=\frac{-3|\delta| \frac{\kappa^{2}}{v_{g}{ }^{3}}}{\left(\delta^{2}+\kappa^{2}\right)^{\frac{5}{2}}} .
$$

The long period grating induced third-order dispersion $\left(\beta_{3}^{g}\right)$ as a function of wavelength $(\lambda)$ is plotted in Figure 6. From this Figure it is observed that the parameter $\beta_{3}^{g}$ remains negative on both branches of the dispersion curve. We find that the behavior of second and third order dispersion is quite different than those of the uniform fiber. $\beta_{2}^{g}$ changes two sides of resonance band centered at the resonance wavelength, whose location can be easily controlled, whereas $\beta_{2}$ for the fiber changes sign at zero dispersion wavelengths and that can be varied only in the range $1.3 \mu \mathrm{m}-1.6 \mu \mathrm{m}$.

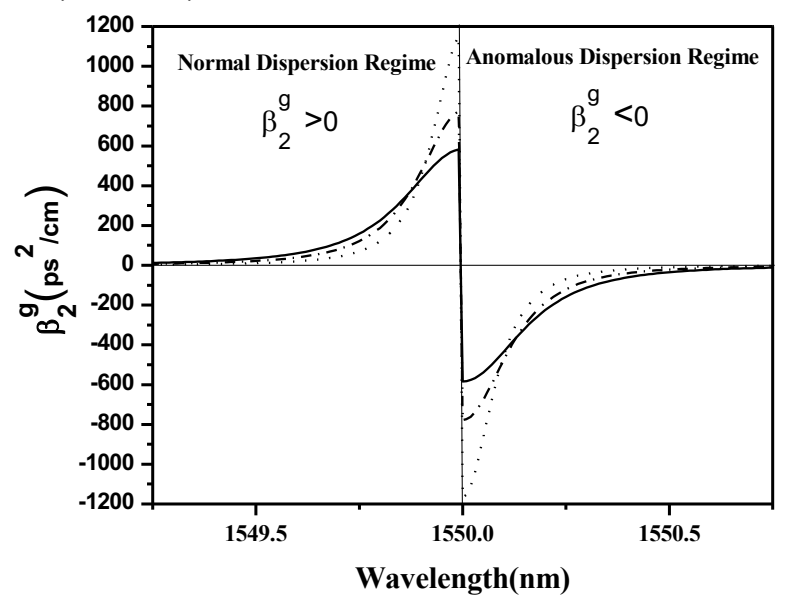

Figure 5: Grating induced group velocity dispersion (GVD) plotted as a function of wavelength $(\lambda)$ for three values of the coupling coefficient $\kappa=2 \mathrm{~cm}^{-1}$ (dotted curve), $\kappa=3 \mathrm{~cm}^{-1}$ (dashed curve) and $\kappa=4 \mathrm{~cm}^{-1}$ (solid curve).

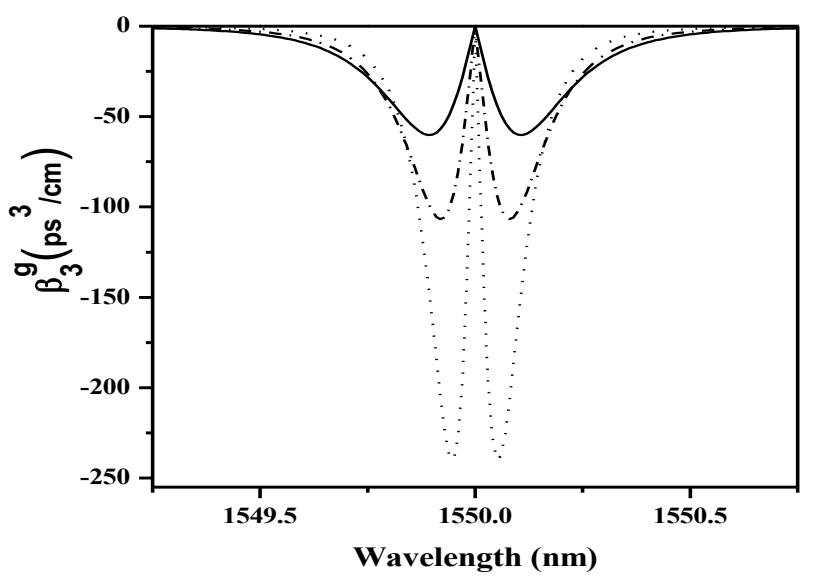

Figure 6: Grating induced third order dispersion plotted as a function of wavelength $(\lambda)$ for three values of the coupling coefficient $\kappa=2 \mathrm{~cm}^{-1}$ (dotted curve), $\kappa=3 \mathrm{~cm}^{-1}$ (dashed curve) and $\kappa=4 \mathrm{~cm}^{-1}$ (solid curve).

The third-order dispersion $\beta_{3}$ is always positive and distorts the optical pulse in optical fiber such that it becomes asymmetric with an oscillatory structure near one of its edges [34]. Thus the second-order dispersion $\beta_{2}$ and thirdorder dispersion $\beta_{3}$ induced pulse broadening in optical fiber can be compensated by the grating induced GVD parameter $\beta_{2}^{g}$ and third order dispersion $\beta_{3}^{g}$. In section $\mathrm{V}$ the phase response of the grating is obtained in Equation (32). Using this Equation the group delay experienced by the frequency component $\omega$ is calculated as [28] 


$$
\tau_{\rho}=\frac{d \phi_{L}}{d \omega}=-\frac{\lambda^{2}}{2 \pi c} \frac{d \phi_{L}}{d \lambda} .
$$

Differentiating Eq.(37) with respect to wavelength $(\lambda)$, we obtained the expression for delay in the form

$$
\tau_{\rho}=-\frac{\lambda^{2}}{2 \pi c}\left[\frac{\left(\frac{\delta}{q}\right) \sec (q L)^{2}\left(\frac{d q}{d \lambda}\right)+\tan (q L) \frac{d}{d \lambda}\left(\frac{\delta}{q}\right)}{1+\left(\frac{\delta}{q}\right)^{2} \tan (q L)^{2}}\right] .
$$

Equation (38) shows the delay response of the grating. It is observed that we can easily vary the delay experienced by the frequency components by proper selection of the grating length and magnitude of induced index change.

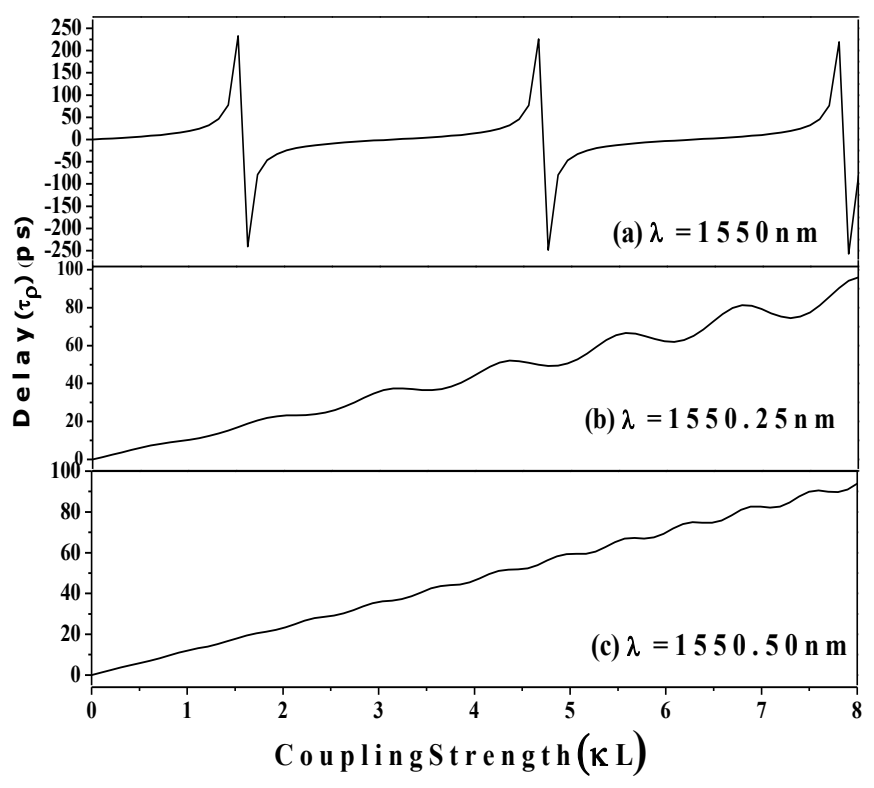

Figure 7: Delay Response of the LPFG as a function of grating coupling strength for different values of incident wavelength (a) $\lambda=1550 \mathrm{~nm}$ (b) $\lambda=1550.25 \mathrm{~nm}$ and (c) $\lambda=1550.50 \mathrm{~nm}$.

Figure 7, illustrate the delay response of the LPFG as a function of coupling strength $\kappa \mathrm{L}$ for three different values of incident wavelength (1) $\lambda=1550 \mathrm{~nm}$ (2) $\lambda=1550.25 \mathrm{~nm}$ and (3) $\lambda=1550.50 \mathrm{~nm}$. The figure (7.a) plotted at incident wavelength $1550 \mathrm{~nm}$, in this sketch we obtain three negative values of delay as (1) $-240.6 \mathrm{ps}$ at value $\kappa \mathrm{L}=1.60$ (2) $248.55 \mathrm{ps}$ at value $\kappa \mathrm{L}=4.76$ and 3 ) $-257 \mathrm{ps}$ at value $\kappa \mathrm{L}=7.90$. It is observed that, at other values of $\kappa L$ the value of delay swing between negative to positive values. Figures (7.b) and (7.c) are plotted for incident wavelength at $1550.25 \mathrm{~nm}$ and $1550.50 \mathrm{~nm}$, respectively. These figures revels that the delay increases in a positive manner as a sinusoidal form or as a stepwise fashion. Thus for the application of dispersion compensation in optical communication system the LPFG can be as a dispersion compensator with physical parameter as: design wavelength $\lambda_{\mathrm{D}}=1550 \mathrm{~nm}$ and the coupling strength as $\kappa \mathrm{L}=1.60,4.76$ and 7.90 . The rate of change of group delay with frequency determines the dispersion experienced by the frequency component. Thus the group velocity dispersion can be calculated as

$$
D_{\rho}=\frac{d \tau_{\rho}}{d \lambda}=-\frac{\lambda}{\pi c} \frac{d \phi_{L}}{d \lambda}-\frac{\lambda^{2}}{2 \pi c} \frac{d^{2} \phi_{L}}{d \lambda^{2}} .
$$

This equation shows the dependency of group velocity dispersion on the wavelength of incident wave inside the grating. The grating induced dispersion coefficient $D_{\rho}$ determines the temporal broadening of the pulse, if $\mathrm{D}_{\rho}<0$ then grating shows the normal (positive) GVD and if $\mathrm{D}_{\rho}>$ 0 grating shows the anomalous (negative) GVD.

\section{Conclusions}

The objective of this work is to provide a complete analytical study of long period fiber grating and to highlights its applications in optical communication system, as an optical filter and a dispersion compensator, we show that, how a long period grating is capable of coupling light from the fundamental guided mode to the cladding modes at specific wavelengths (resonance wavelengths) leading to the formation of so called rejection bands in the transmission. The role of physical parameters such as grating length and induced refractive index change of grating on filter characteristics, dispersion properties and group delay spectra are also analysed. It is envisaged that the waveguide based LPFG should provide a useful approach to the design of a wide range of integrated-optical devices, including wavelength-tunable filters, dispersion compensator, switches, and environmental sensors without compromising much with the numerical accuracy.

\section{Acknowledgements}

The authors are thankful to Dr. Pratima Sen of Devi Ahilya Vishwavidyalaya, Indore for her critical comments and suggestions. One of the authors Santosh Pawar would like to acknowledge the MPCST, Bhopal for financially support of the project.

\section{References}

[1] A. M. Vengaskar, P. J. Lemaire, V. Bhatia, T. Erdogan, J. E. Sipe, Long-period fiber gratings as band rejection filters, J. Lightwave Techno. 14: 58-65, 1996.

[2] R. Singh, E. K. Sharma, Gain flattening by long period gratings in erbium doped fibers, Opt. Comm. 240:123132, 2004.

[3] D. B. Stegall, T. Erdogan, Dispersion control with use of long-period fiber gratings, J. The Optical Society of America A 17:304-312, 2000.

[4] J. F. Huang, Y. J. He, Y. L. Lo, Bandwidth analysis of long-period fiber grating for high-order cladding mode and its application to optical add-drop multiplexer, Opt. Eng. 45:125001.1-125001.8, 2006.

[5] B. Ortega, L. Dong, W. F. Liu, J. P. de Sandro, L. Reekie, S. I. Tsypina, R. I. Laming, High-performance optical fiber polarizers based on long-period gratings in 
birefringent optical fibers, IEEE, Photon.Technol. Lett. 9: 1370-1372, 1997.

[6] V. Bhatia, A. M. Vengsarkar, Optical fiber long-period grating sensors, Opt. Lett. 21:692-694, 1996.

[7] J. Blows, Y. D. Tang, Gratings written with tripled output of Q-switched Nd:YAG laser, IEEE, Electron. Lett. 36:1837-1839, 2000.

[8] M. Fujumaki, Y. Ohki, J. L. Brebner, S. Roorda, Fabrication of long-period optical fibre gratings by use of ion implantation, Opt. Lett. 25: 88-90, 2000.

[9] Y. Kondo, K. Nouchi, T. Mitsuyu, M. Watanabe, P. Kazansky, K. Hirao, Fabrication of long-period fibre gratings by focused irradiation of infra-red femtosecond laser pulses, Opt. Lett. 24:646-648, 2000.

[10] D. Davis, T. K. Gaylord, E. N. Glytsis, S. G. Kosinski, S. C.Mettler, A. M.Vengsarkar, Long-period fibre grating fabrication with focused $\mathrm{CO} 2$ laser beams, IEEE, Electron. Lett. 34:302-303, 1998.

[11] E. M. Dianov, V. I. Karpov, A. S. Kurkov, M. V. Grekov, Long period fibre gratings mode-field converters fabricated by thermos diffusion in phosphosilicate fibres, Proc. 24th ECOC, Madrid: Telefonica Espana, pp. 1-3, 1998.

[12] C. S. Kim, Y. Han, B. H. Lee, W. T. Han, U. C. Paek, $Y$. Chung, Induction of the refractive index change in B-doped optical fibers through relaxation of the mechanical stress, Opt. Comm. 185:337-342, 2000.

[13] G. Rego, O. Okhotnikov, E. Dianov, V. Sulimov, Hightemperature stability of long-period fibre gratings using an electric arc, IEEE, J. Lightwave Technol. 19: 1574$1579,2001$.

[14] T. Allsop, K. Kalli, K. Zhou, M. Dubov, D. J. Webb, I. Bennion, Long period gratings written into a photonic crystal fibre by a femtosecond laser as directional bend sensors, Optics Comm. 281: 5092-5096, 2008.

[15] D. Kowal, G. Statkiewicz-Barabach, P. Mergo, W. Urbanczyk, Microstructured polymer optical fiber for long period gratings fabrication using an ultraviolet laser beam, Optics Letter 39:2242-2245, 2014.

[16] D. Pudo, Eric C. Magi, B. J. Eggleton, Long-period gratings in chalcogenide fibers, Optics Express 14:3763-3766, 2006.

[17] I Kag Hwang, S. H. Yun, B. Y. Kim, Long-period fiber gratings based on periodic microbends, Optics Letter 24,1263-1265, 1999.

[18] H. E. Engan, B. Y. Kim, J. N. Blake, H. J. Shaw, Propagation and optical interaction of guided acoustic waves in two-mode optical fibers, IEEE J. Lightwave Techno. 6:428-436, 1988.

[19] Y. Zhao, J. C. Palais, Simulation and characteristics of long-period fibre Bragg grating coherence spectrum, IEEE J. Lightwave Techno. 16:554-561, 1998.

[20] I. Navruz, A. Altuncu, Optimization of phase shifted long-period fiber gratings for multiband rejection filters, IEEE J. Lightwave Techno. 26: 2155-2161, 2008.
[21] H. Jeong, K. Oh, Enhancement of free spectral range of resonance peaks of a long period fiber grating by controlling material dispersion of cladding modes, Optics Comm. 199:103-110, 2001.

[22] Y. Bai, K. S. Chiang, Transmission characteristics of long period waveguide grating couplers Technology, IEEE J. Lightwave Techno. 21: 3399-3405, 2003.

[23] H. J. Patrick, A. D. Kersey, F. Bucholtz, Analysis of the response of long period fiber gratings to external index of refraction, IEEE J. Lightwave Techno. 16:16061612, 1998.

[24] K. S. Chiang and Q. Liu, Long-period gratings for application in optical communications, Proc. International Symposium on Advances and Trends in Fiber Optics and Applications (ICOCN/ATFO), Chengdu, China, pp. 128-133, 2006.

[25] V. Rastogi, K. S. Chiang, Long-period gratings in planar optical waveguides, Appl. Opt. 41:6351-6355, 2002.

[26] O. Deparis, R. Kiyan, O. Pottiez, and M. Blondel, Bandpass filters based on $\pi$-shifted long-period fiber gratings for actively mode-locked erbium fiber lasers, Opt. Lett., 26:1239-1241, 2001.

[27] Y. Gu, K. S. Chiang, Effects of average index variation in apodized long-period fiber gratings, Photonic Sensors 32:102-111, 2013.

[28] M. Das, K. Thyagarajan, Dispersion compensation in transmission using uniform long period fiber gratings, Optics Comm. 190:159-163, 2001.

[29] T. Erdogan, Fiber Grating Spectra, IEEE J. Lightwave Technol., 15:1277-1294, 1997.

[30] A. W. Snyder, J. D. Love, Optical Waveguide Theory Chapman and Hall Publishers, 1983.

[31] H. Kogelnik, Filter response of non-uniform almost periodic structures, J. Bell Sys. Tech., 55:109-126,1976.

[32] D. Marcuse, Theory of Dielectric Optical waveguides, Academic Publishers, New York, 1991.

[33] L. Poladian, Resonance mode expansions and exact solutions for nonuniform gratings, Phys. Rev. E 54:2963-2975, 1996.

[34] G. P. Agrawal, Application of Nonlinear Fiber optics, Academic Press, San Diego, 2001.

[35] J. N. Kutz, B. J. Eggleton, J. B. Stark, R. E. Slusher, Nonlinear Pulse Propagation in Long-Period Fiber Gratings: Theory and Experiment, IEEE Sel. Topics Quantum Electron., 3:1232-1245, 1997.

[36] A. Carballar, M. A. Muriel, Phase Reconstruction from Reflectivity in Fiber Bragg Gratings, IEEE J. Lightwave Techno. 15:1314-1322, 1997.

[37] V. Jain, S Pawar, S Kumbhaj, P K Sen, Analysis of dispersion characteristics of long period fiber grating, Journal of Physics: Conference Series 755: 012057012061, 2016. 